\title{
Reverse Doppler effect in left-handed travelling-wave field-effect transistors
}

\author{
Koichi Narahara ${ }^{\text {a) }}$ \\ Graduate School of Science and Engineering, Yamagata University, \\ 4-3-16 Jonan, Yonezawa, Yamagata 992-8510, Japan \\ a)narahara@yz.yamagata-u.ac.jp
}

\begin{abstract}
We investigate left-handed ( $\mathrm{LH})$ travelling-wave fieldeffect transistors (TWFETs) to observe the reverse Doppler effect. Due to the nonlinearity, a TWFET can support shock fronts that can reflect incoming waves. By introducing the composite right/left-handed line structure to a TWFET, it enables to support LH waves. Because the shock front functions as a moving reflection wall to the incoming $\mathrm{LH}$ waves, we can expect the reverse Doppler effect to cause in an LH TWFET. After explaining the structure and fundamental properties, we report the reverse Doppler effect observed experimentally in the devise.
\end{abstract}

Keywords: reverse Doppler effect, left-handed waves, traveling-wave field-effect transistors

Classification: Electron devices, circuits, and systems

\section{References}

[1] V. G. Veselago, "Electrodynamics of substances with simultaneously negative electrical and magnetic properties," Sov. Phys. USP., vol. 10, pp. 509$517,1968$.

[2] S. H. Lee, C. M. Park, Y. M. Seo, Z. G. Wang, and C. K. Kim, "Composite acoustic medium with simultaneously negative density and modulus," Phys. Rev. Lett., vol. 104, 054301, 2010.

[3] N. Seddon and T. Bearpark, "Observation of the inverse Doppler effect," Science, vol. 302, pp. 1537-1540, 2003.

[4] Y. Yuan, Y. Feng, and T. Jiang, "Observation of reverse Doppler effect in a composite right/left-handed transmission line," Proc. China-Japan Microwave Conf., pp. 1-4, 2011.

[5] E. J. Read, M. Soljacic, and J. D. Joannopoulos, "Reversed Doppler effect in photonic crystals," Phys. Rev. Lett., vol. 91, 133901, 2003.

[6] G. W. McIver, "A traveling-wave transistor," Proc. IEEE, vol. 53, pp. 1747-1748, 1965.

[7] K. Narahara, "Development of shock waves in traveling-wave field-effect transistors," J. Appl. Phys., vol. 112, 084914, 2012.

[8] C. Caloz and T. Itoh, Electromagnetic metamaterials: transmission line theory and microwave applications, Wiley, New York, 2006.

[9] K. P. Hadeler, "Hyperbolic traveling fronts," Proc. Edinburgh Society, vol. 31, pp. 89-97, 1988. 


\section{Introduction}

Since the theoretical study by Veselago in 1968 [1], left-handed (LH) media have attracted attentions as platforms to exhibit several exotic electromagnetic phenomena. In such media, frequency is expected to decrease by the reflection with the approaching wall unlike the normal media having positive refractive index. This phenomenon is presently called the reverse Doppler effect. Several platforms have been reported to observe this effect for acoustic [2], radio frequency [3, 4], and optical [5] waves. Recently, we found that travelling-wave field-effect transistors (TWFETs), originally introduced by McIver in 1965 [6], can support shock waves by applying properly designed decreasing and increasing kink-like voltages to the drain and gate lines, respectively [7]. The shock fronts accompany the finite drain currents, so that the incoming voltage waves are partially screened and reflected. Moreover, we introduced composite right/left-handed (CRLH) transmission line structure [8] to the gate and drain lines of a TWFET, being compatible with the development of shocks. With the advent of resulting LH waves, we successfully observed the reverse Doppler effect by time-domain measurements of their collision with a shock front.

\section{Fundamental properties of LH TWFETs}

Figure 1 shows the cell structure of an LH TWFET we investigated. The series section of gate (drain) line consists of the per-unit-cell inductance $L_{R G(D)}$ and capacitance $C_{L G(D)}$. On the other hand, the shunt section of the gate

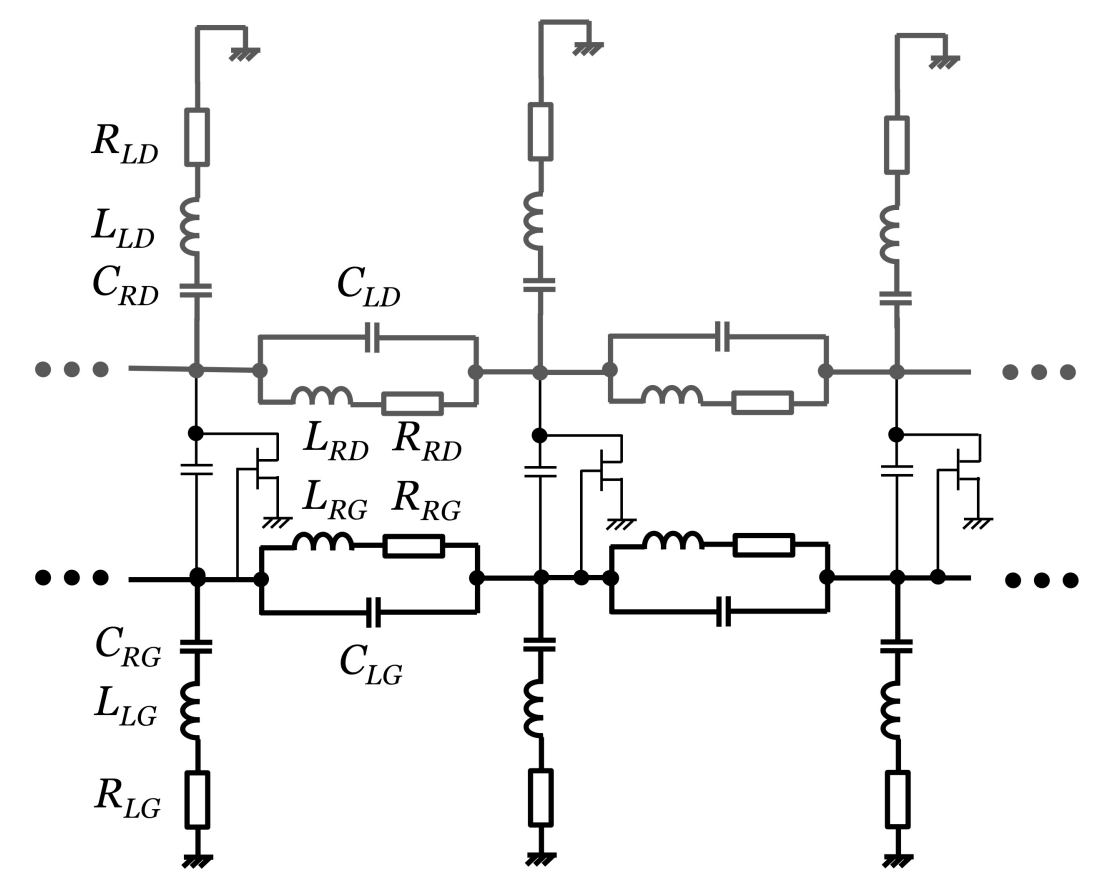

Fig. 1. Structure of LH TWFET we investigated. Black and grey elements represent the gate and drain lines, respectively. The lines are coupled via the mutual capacitors and FETs. 
(drain) line consists of the per-unit-cell inductance $L_{L G(D)}$ and capacitance $C_{R G(D)}$. Moreover, $R_{R G(D)}$ and $R_{L G(D)}$ represent the parasitic resistance of $L_{R G(D)}$ and $L_{L G(D)}$, respectively. Unlike the ordinary CRLH line structure, $C_{L G(D)}$ is put in parallel with $L_{R G(D)}$, and $L_{L G(D)}$ is put in series with $C_{R G(D)}$, in order to support baseband right-handed (RH) waves. The gate and drain lines are coupled by the mutual capacitance $C_{m}$ and FET. For simplicity, we consider the case when both lines have identical values of perunit-cell elements, i.e., we set $L_{R G}=L_{R D}=L_{R}, R_{R G}=R_{R D}=R_{R}, C_{R G}=$ $C_{R D}=C_{R}, L_{L G}=L_{L D}=L_{L}, R_{L G}=R_{L D}=R_{L}$, and $C_{L G}=C_{L D}=C_{L}$.

Figure 2 shows the dispersion relationship of the line having $C_{R}=C_{L}$ $=470 \mathrm{pF} /$ cell, $L_{R}=L_{L}=100 \mu \mathrm{H} /$ cell, and $C_{m}=47 \mathrm{pF} /$ cell. A band-gap separates the frequencies allowing traveling waves into two. There are two almost degenerate RH modes in the baseband frequencies. Each has either the even- or odd-polarity voltage fraction between the gate and drain lines. Utilizing the odd-polarity one, shock waves can develop. On the other hand, at the higher frequencies than the band-gap, two modes can carry LH waves at most. The solid and dashed curves correspond to the even- and oddpolarity LH modes, respectively.

For shock wave formation, the falling and rising kink-like voltages are simultaneously applied at the first cells of the drain and gate lines, respectively, such that the voltage slope of the gate line must cross the FET threshold voltage $V_{t h}$. Moreover, the drain voltage finally attained must be set close to zero such that the FET drain-source current $I_{d s}$ becomes zero after the transients. On the other hand, the initial gate voltage is set less than $V_{t h}$, whereas the initial drain voltage is set to a finite positive value that determines the shock

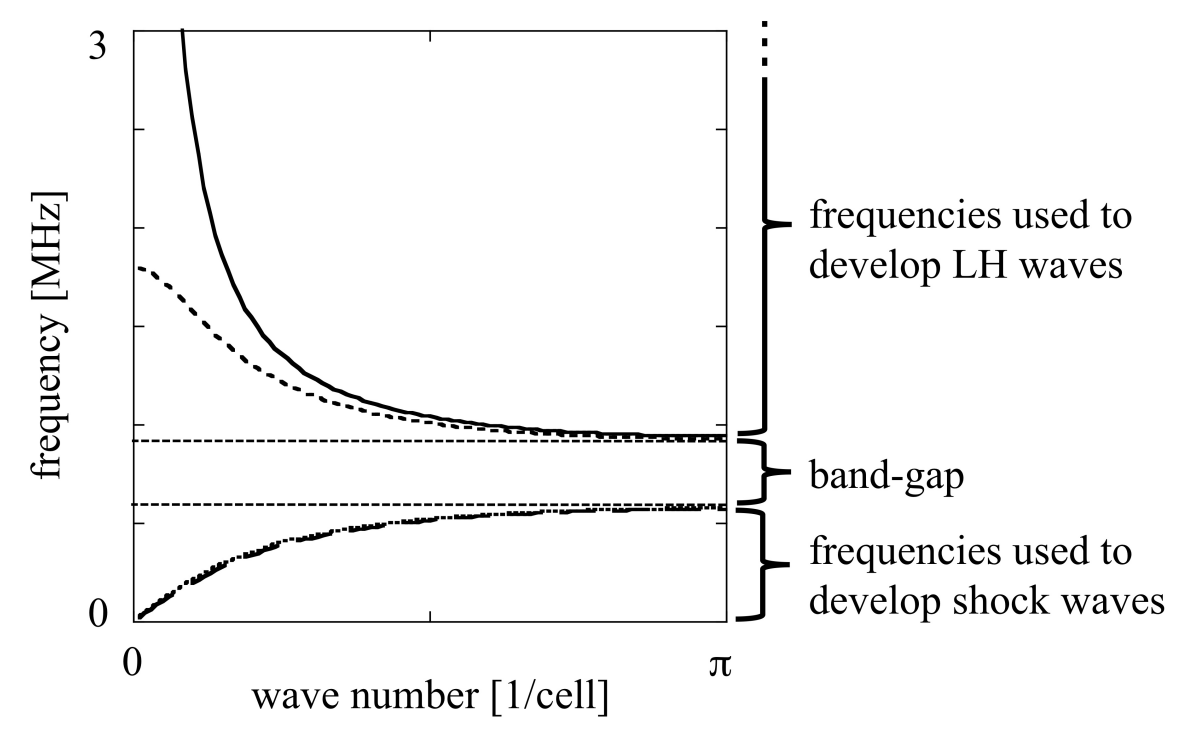

Fig. 2. Dispersion relationship of measured LH TWFET. Four curves represent the travelling-wave modes. In order to show clearly the dispersion around the band-gap, frequencies are shown up to $3 \mathrm{MHz}$. Note that the solid curve approaches the vertical axis monotonically for higher frequencies. 
amplitude. The voltage slopes on the gate and drain lines are generally carried by the baseband odd-polarity mode and travel on the lines without temporal/spatial deviation. It is then shown that the voltage slopes in a TWFET are governed by the nonlinear hyperbolic equation [9], such that they evolve into shock waves when $C_{R} R_{R}+L_{R} G(V)$ becomes zero at some drain voltage $V$, where $G(V)$ represents the dynamical drain conductance defined by $G(V)=d I_{d s}(V) / d V[7]$.

\section{Experiments}

We carried out the time-domain measurements of a 200-cell LH TWFET built on a standard breadboard. The transistors used were of the type Toshiba 2SK30A junction FET, whose typical $V_{t h}$ was $-1.5 \mathrm{~V}$. Every inductance was implemented using $100 \mu \mathrm{H}$ TDK SP0508 inductor. Moreover, $C_{R}$ and $C_{L}$ were implemented with $470 \mathrm{pF}$ TDK FK18C0G1 capacitors. The same type capacitor was used to implement $C_{m}$ with $47 \mathrm{pF}$ capacitance. Signals along the test LH TWFET were detected by an Agilent 1134A active probe and monitored in the time domain using an Agilent DSO90254A oscilloscope.

Figure 3 shows the measured shock front. Ten temporal waveforms are shown, recorded at different cells on the drain line with 10-cell increments. The 200th cells of the gate and drain lines were supplied with -2.5 and $2.0 \mathrm{~V}$ DC sources, respectively. On the other hand, the 1st cells of the gate and drain lines were fed by the increasing and decreasing voltage pulses generated by a two-channel arbitrary waveform generator NF WF1974. The durations of voltage slopes were set to $10 \mu \mathrm{s}$. In Fig. 3, we can see that the voltage slope becomes significantly steeper at the very early stage of propagation, and its shape is almost preserved.

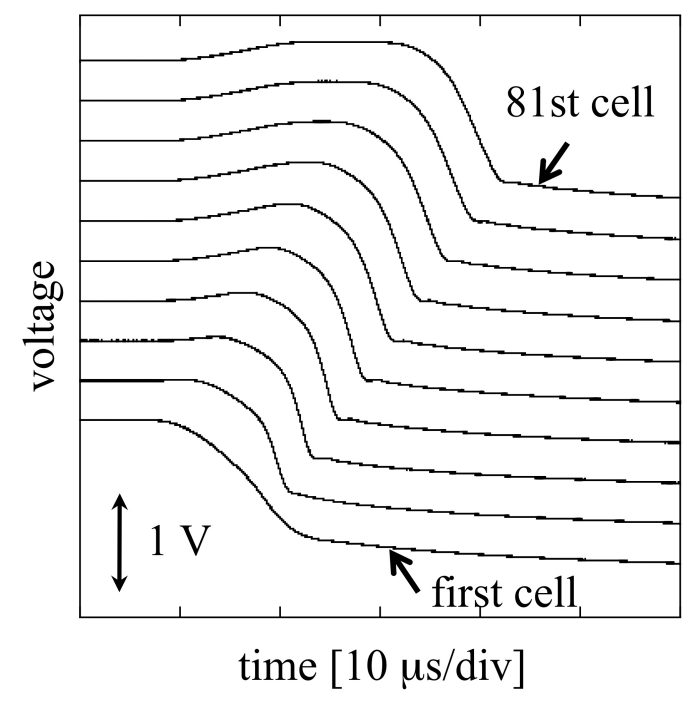

Fig. 3. Measured shock front. Temporal waveforms recorded at ten different cells on the drain line are shown. The increments are 10 cells. The bottom and top represent ones recorded at the 1st and the 81st cells, respectively. 
We then fed the 200th cells with the sinusoidal waveforms using another NF WF1974 waveform generator phase-synchronized with the first one. The waveforms applied at the gate and drain lines were set to be identical, such that they were carried by the even-polarity LH mode represented by the solid curve in Fig. 2. Suppose that a sinusoidal wave having the angular frequency $\omega_{i}$ is reflected by an approaching wall with the velocity $v_{s}$. As shown in [3], the angular frequency of the reflected sinusoidal wave $\omega_{r}$ is given in LH media by

$$
\omega_{r}=\frac{v_{\phi}\left(\omega_{i}\right)-v_{s}}{v_{\phi}\left(\omega_{r}\right)+v_{s}} \omega_{i}
$$

where the function $v_{\phi}(\omega)$ represents the phase velocity of the even-polarity LH mode at the angular frequency $\omega$ given by

$$
v_{\phi}(\omega)=\frac{\omega}{\cos ^{-1}\left[\frac{2-\left\{2 C_{L} L_{R}+C_{R}\left(2 L_{L}+L_{R}\right)\right\} \omega^{2}+2 C_{L} C_{R} L_{L} L_{R} \omega^{4}}{2\left(C_{R} L_{L} \omega^{2}-1\right)\left(C_{L} L_{R} \omega^{2}-1\right)}\right]} .
$$

Unlike RH media, $\omega_{r}$ becomes smaller than $\omega_{i}$ by the approaching reflection wall.

Figure 4 summaries the reverse Doppler effect measured in the test LH TWFET. The temporal separation between the shock and sinusoidal waves was tuned for them to start to collide at around the 150th cell. Figure 4 (a)

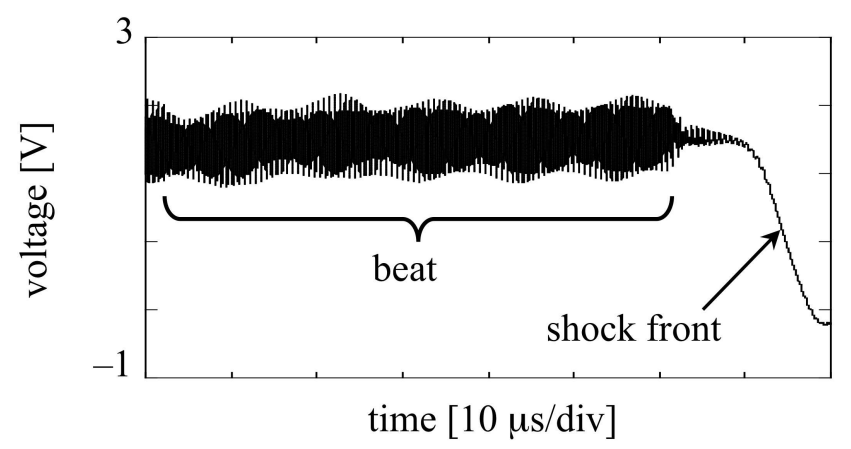

(a)

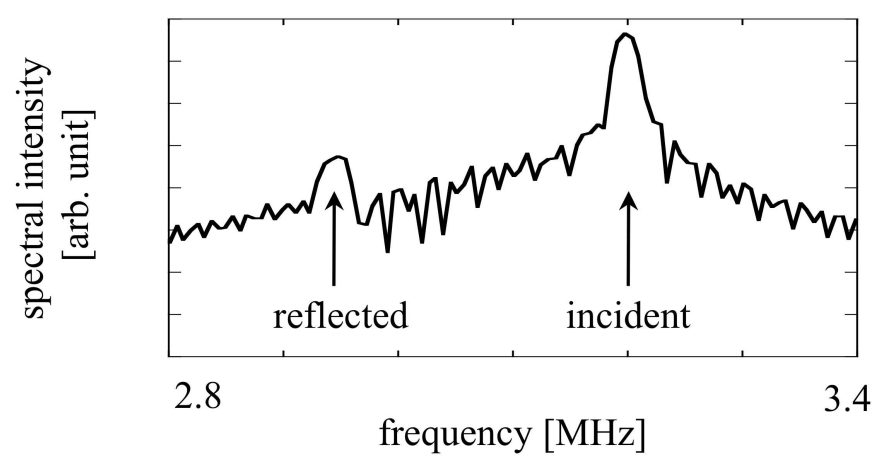

(b)

Fig. 4. Reverse Doppler effect in measured LH TWFET. (a) The temporal waveform recorded at the 161st cell on drain line and (b) the spectral intensity obtained by Fourier transform of the waveform in Fig. 4 (a). 


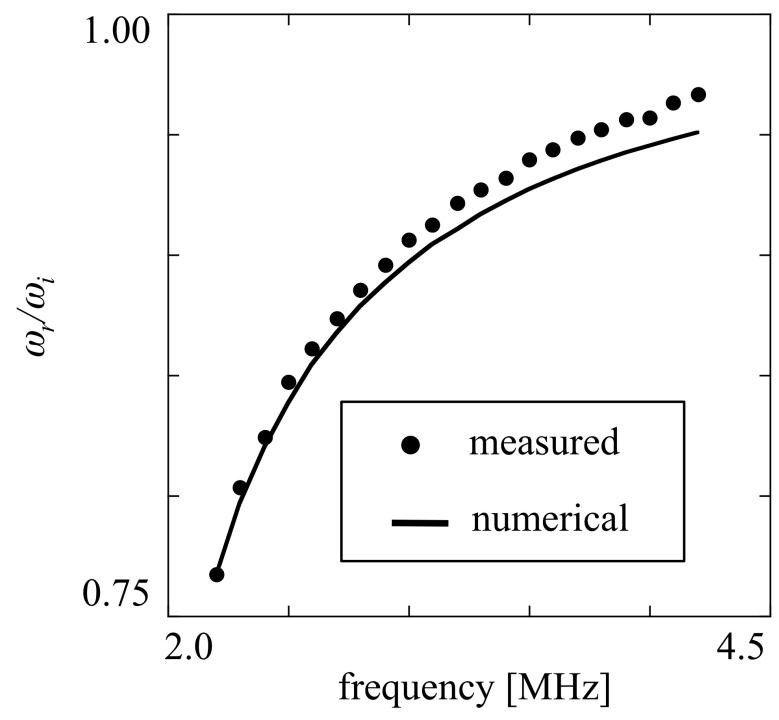

Fig. 5. Fraction between incident and reflected frequencies. Circles and solid curve show measured and calculated fractions, respectively.

shows the temporal waveform monitored at the 161st cell on the drain line for the $3.2-\mathrm{MHz}$ sinusoidal waves. Because of the difference in incident and reflected frequencies, the observed superposed waveform is supposed to exhibit the beat, which is clearly observed in Fig. 4 (a). The spectral intensity calculated by the numerical Fourier transform is shown in Fig. 4(b). Both of the peaks corresponding to the incident and reflected waves were successfully detected. Note that the frequency of the reflected wave was peaked at $2.95 \mathrm{MHz}$, being smaller than the incident one.

Similar evaluations were carried out for other incident frequencies. The resulting fraction between the reflected and incident frequencies $\omega_{r} / \omega_{i}$ is plotted for several different values of $\omega_{i}$ in Fig. 5. Circles show the measured fractions. For all the measuring incident frequencies, $\omega_{r}$ becomes smaller than $\omega_{i}$. The frequency fraction predicted by Eqs.(1) and (2) for setting $v_{s}$ to $3.5 \times 10^{6} \mathrm{cell} / \mathrm{s}$ is shown by the solid curve, which well explains the measured result. It is thus concluded that the reverse Doppler effect was verified experimentally in an LH TWFET.

\section{Conclusion}

With shock formation, an LH TWFET becomes a good platform to exhibit the reverse Doppler effect. The devise only utilizes FETs as the sources of nonlinearity, which can potentially operate at high frequencies including millimetre-waves. 